\title{
Identification of autoantibody against fatty acid synthase in hepatocellular carcinoma mouse model and its application to diagnosis of HCC
}

\author{
CHANG-KYU HEO ${ }^{1,7}$, MI-KYUNG WOO ${ }^{1}$, DAE-YEUL YU ${ }^{2}$, JU YEON LEE ${ }^{3}$, \\ JONG SHIN YOO ${ }^{3}$, HYANG SOOK YOO ${ }^{1}$, JEONG HEON KO ${ }^{1}$, JIN-MAN KIM ${ }^{4}$, \\ JONG YOUNG CHOI ${ }^{5}$, IN GYU KIM ${ }^{6}$, SANG GI PAIK ${ }^{7}$ and EUN-WIE CHO ${ }^{1}$
}

\begin{abstract}
${ }^{1}$ Daejeon-KRIBB-FHCRC Research Cooperation Center, Korea Research Institute of Bioscience and Biotechnology (KRIBB); ${ }^{2}$ Department of Human Genomics, KRIBB, Daejeon 305-860; ${ }^{3}$ Division of Proteome Research, Korea Basic Science Institute, Daejeon 305-333; ${ }^{4}$ Department of Pathology, College of Medicine, Chungnam National University, Daejeon 301-131; ${ }^{5}$ Department of Internal Medicine, College of Medicine, the Catholic University of Korea, Seoul 137-040; ${ }^{6}$ Department of Radiation Biology, Environmental Radiation Research Group, Korea Atomic Energy Research Institute, Daejeon 305-600; ${ }^{7}$ Department of Biology, School of Biosciences and Biotechnology, and the Institute of Biotechnology, Chungnam National University, Daejeon 305-764, Korea
\end{abstract}

Received November 10, 2009; Accepted February 22, 2010

DOI: 10.3892/ijo_00000631

\begin{abstract}
Autoantibodies, which are generated by immune system recognizing the presence of the abnormal tumorassociated antigens, are promising biomarkers for early detection of tumors. Recently, we established a B cell hybridoma pool derived from $\mathrm{H}-$ ras $12 \mathrm{~V}$ transgenic mouse, a typical hepatocellular carcinoma model, as a source of tumorassociated autoantibodies without using any extracellular antigens and have characterized the specific target antigens against them. K1 autoantibody, one of them, was investigated in this study and its target antigen was identified by mass spectrometric analysis as fatty acid synthase (FASN), an important oncogenic protein. Moreover, a specific mimotope against K1 autoantibody was screened from the cyclic random hepta-peptide phage library and, using it as a coating antigen for ELISA, we could distinguish patients with hepatocellular carcinoma (HCC) vs. normal subjects with $96.55 \%$ sensitivity and $100 \%$ specificity. These results imply that anti-FASN autoantibody is induced in patients with HCC and detection of anti-FASN autoantibody can be used for the diagnosis of HCC.
\end{abstract}

Correspondence to: Dr Eun-Wie Cho, Daejeon-KRIBB-FHCRC Research Cooperation Center, Korea Research Institute of Bioscience and Biotechnology, 111 Gwahangno, Yuseong-gu, Daejeon 305-860, Korea

E-mail: ewcho@kribb.re.kr

Key words: tumor-associated autoantibody, H-ras $12 \mathrm{~V}$ mouse, fatty acid synthase, hepatocellular carcinoma

\section{Introduction}

Currently available serum markers are based on the measurement of tumor antigens, such as $\alpha$-fetoprotein (AFP), prostatespecific antigen (PSA), and cancer antigen CA125. However, no single marker has been recognized as a true cancer marker due to lack of sensitivity and specificity. To overcome the limitations of these tumor antigen biomarkers, alternative attempts are sought for the development of cancer biomarkers based on the detection of circulating autoantibodies against tumor-associated antigens in patient sera (1).

A variety of techniques to discover novel tumor-associated autoantigens have been developed, such as SEREX (serological analysis of recombinant cDNA expression libraries), two-dimensional electrophoresis/Western blotting of tumor cell lysates, panning of a phage display library constructed using tumor cell cDNA library, and protein microarray (2). These techniques were expected to be powerful, which present thousands of candidate antigens at once to allow analysis of many kinds of autoantibodies in patient sera. But patient sera are mixtures of hundreds of autoantibodies and the amount of each autoantibody is not comparable to another, which makes the discovery of autoantigens biased toward the most abundant ones. For these reasons, tumor-associated antigens discovered by these techniques are not so diverse and useful, despite the expectation that hundreds of tumor-associated antigens and specific autoantibodies against them are present in patient sera.

To identify tumor-associated autoantibodies of hepatocellular carcinoma (HCC), we constructed B-cell hybridoma pool using splenocytes derived from H-ras12V HCC mouse model (3) and the stable hybridoma clones that produce antibodies reactive to HCC cells were selected. Several hundreds of autoantibody-secreting B-cell lines were established and we have analyzed the specific antigens against these antibodies. In 
this study, the target antigen against $\mathrm{K} 1$ autoantibody was analyzed and revealed as fatty acid synthase (FASN), a wellknown oncogenic protein (4). In addition, a specific mimotope of K1 autoantibody was screened from a cyclic random heptapeptide phage library and, when using it as a coating antigen for ELISA, we could distinguish patients with HCC from normal subjects with $96.55 \%$ sensitivity and $100 \%$ specificity. These results indicate that anti-FASN autoantibody is induced in HCC patient sera and the detection of anti-FASN autoantibody can be useful for the diagnosis of HCC.

\section{Materials and methods}

Cell lines and serum samples. The cell lines were obtained from American Type Culture Collection (ATCC) and cultured in DMEM or RPMI-1640 medium containing 10\% fetal bovine serum. All cell lines originated from humans, except Hepa$1 \mathrm{c} 1 \mathrm{c} 7$, which is a mouse hepatoma cell line. Human HCC serum samples were collected from Catholic Hospital (Seoul). Normal serum samples were collected from volunteers or patients without cancer. Serum samples were kept at $-70^{\circ} \mathrm{C}$ until use.

Preparation of monoclonal autoantibodies reactive to hepatoma cells. Splenocytes from H-ras $12 \mathrm{~V}$ transgenic mice (3) at about 10 months old were used for the construction of B-cell hybridoma cell pool producing tumor-associated autoantibodies. Cell fusion and selection of B-cell hybridoma were performed as described previously (5). The reactivity of antibodies produced by these B-cell clones was determined by intracellular staining of HepG2 or Hepa-1c1c7 cells with each autoantibody, followed by flow cytometric analysis as described below. The isotypes of each autoantibody were determined using an isotyping kit (Pierce). For preparation of K1 autoantibody, ascites fluid was produced and antibody was purified using protein L-agarose (Pierce).

Flow cytometric analysis of autoantibodies. Cells were fixed and permeabilized with BD cytoperm/cytofix solution (BD), followed by a incubation with primary antibody solution (hybridoma cell cultured media or purified antibody) at $4^{\circ} \mathrm{C}$ for $40 \mathrm{~min}$. Cells were washed and stained with anti-mouse Ig goat $\left(\mathrm{Fab}^{\prime}\right)_{2}$-RPE (Dako). The stained cells were analyzed by FACScalibur (BD) and obtained data were analyzed using CellQuest software (BD). When determining whether the autoantibody-mimotope phage can compete with target cellular antigen for the antibody binding, primary antibodies were preincubated with each phage at room temperature for $60 \mathrm{~min}$.

Preparation of whole cell lysates and Western blot analysis. Cells were lysed in RIPA buffer (50 mM Tris- $\mathrm{HCl}, \mathrm{pH} 7.4$, $150 \mathrm{mM} \mathrm{NaCl}, 0.5 \%$ sodium deoxycholate, $1 \% \mathrm{NP}-40,0.1 \%$ SDS) supplemented with $1 \mathrm{mM}$ EDTA, protease inhibitors (Roche), and phosphatase inhibitors (Roche). Equal amounts of protein $(50 \mu \mathrm{g})$ were resolved by SDS-PAGE and transferred onto a polyvinylidenedifluoride membrane (Millipore). The membranes were probed with $\mathrm{K} 1$ antibody and HRPlinked anti-mouse Ig GAM antibody (Abcam). Positive bands were detected by chemiluminescence (GE).

Immunofluorescence microscopy. Cells plated on 18x18-mm glass coverslips in 6-well plates were treated with BD cytofix/ cytoperm solution at $4^{\circ} \mathrm{C}$ for $40 \mathrm{~min}$. Fixed/permeabilized cells were incubated with $\mathrm{K} 1$ antibody diluted in BD cytoperm/ wash solution $(5 \mu \mathrm{g} / \mathrm{ml})$. After an 1 -h incubation at $4^{\circ} \mathrm{C}$, cells were washed and incubated with FITC-conjugated antimouse immunoglobulin $\mathrm{F}\left(\mathrm{ab}^{\prime}\right)_{2}$ antibody at $4^{\circ} \mathrm{C}$ for $1 \mathrm{~h}$. Coverslips were mounted with Vectshield medium containing DAPI (Vector Laboratories) and analyzed using a Zeiss LSM510 Meta microscope (Carl Zeiss MicroImaging).

Panning of the phage library against K1 antibody. For the selection of the mimotope specific to K1 autoantibody, the phage display random cyclic peptide library, Ph.D. - C7C $\mathrm{C}^{\mathrm{TM}}$ (New England Biolabs), was used following the manufacturer's instructions. Briefly, $2 \times 10^{11}$ phage virions were incubated with $300 \mathrm{ng}$ K1 antibody, followed by affinity capture of antibody-phage complexes onto protein L-agarose beads. Bound phages were eluted and used for determination of the phage titer or amplification. The amplified phages were precipitated using 20\% PEG/2.5 M NaCl and used for the next round of panning. Panning was repeated four times and sequencing of selected mimotope phages was performed following the manufacturer's instructions.

Phage ELISA. The ELISA plate (Maxisorp; Nunc) was coated with selected phage $\left(10^{10} \mathrm{pfu} / \mathrm{well}\right)$ in $0.1 \mathrm{M}$ sodium carbonate buffer ( $\mathrm{pH}$ 8.6). After the wells were blocked with proteinfree blocking buffer (Pierce), primary antibody solution (100 ng purified mAb/100 $\mu \mathrm{l}$ blocking buffer) was added and incubated at room temperature for $2 \mathrm{~h}$. For the secondary reagent, HRP-linked anti-mouse immunoglobulin GAM antibody (Sigma; 1:2000 diluted in blocking buffer) was used. Tetramethylbenzidine-based substrate solution (Pierce) was used for the color development. For evaluation of the effect of reducing disulfide bonds on peptide antigenicity, cyclic phages were reduced and alkylated and then coated onto ELISA plates following the methods of Rojas et al (6). For the detection of reactivity of patient's sera to specific phages, human sera (1:1000 diluted in protein-free blocking buffer) pre-adsorbed with empty phage $\left(10^{10} \mathrm{pfu} / 100 \mu \mathrm{l}\right)$ and ER2738 extract $(20 \mu \mathrm{g} /$ $100 \mu \mathrm{l})$ were used and detected by HRP-conjugated antihuman immunoglobulin GAM antibody (1:2000 diluted in protein-free blocking buffer). Empty M13 phage without a peptide insert was used as control coating antigen. ELISA results were evaluated by a receiver operating characteristics (ROC) curve using Prism 5 (GraphPad Software).

Immunoprecipitation of target protein and mass analysis. For the immunoprecipitation of target protein against K1 autoantibody, purified K1 antibody was conjugated to AminoLink plus coupling resin (Pierce) following the manufacturer's instructions. Then, Hepa-1c1c7 cell lysates prepared in RIPA buffer $(2 \mathrm{mg} / \mathrm{ml})$ were incubated with $\mathrm{K} 1$ antibody-conjugated beads overnight at $4^{\circ} \mathrm{C}$. Elution was performed twice with $0.5 \mathrm{mM}$ K1p7 peptide (Table I; Peptron) dissolved in PBS and eluate was concentrated using Speedvac. The concentrate was run on $8-10 \%$ SDS-PAGE and subjected to immunoblotting or Coomassie staining. The bands corresponding to those probed by $\mathrm{K} 1$ antibody were excised and used for in-gel digestion. Protein identification was performed by using Nano-LC/ESIMS/MS as described by Lee et al (7). 
siRNA transfection. HepG2 cells were transfected with siRNA against FASN (Bioneer) by using Lipofectamine 2000 transfection reagent (Invitrogen) following the manufacturer's instructions. RT-PCR or Western blotting was performed at $72 \mathrm{~h}$ after transfection. The sequences of siRNA against FASN are as following; sense: 5'-CUGUAACUGUCAGUGUACA (dTdT)-3', antisense: 5'-UGUACACUGACAGUUACAG $(\mathrm{dTdT})-3^{\prime}$.

RT-PCR analysis. Total RNA was extracted from cells using Qiagen RNA extraction kit (Qiagen) and the first-strand cDNA was synthesized using Superscript III (Invitrogen). RT-PCR for FASN was performed using the following primer pairs: forward primer: 5'-CCCCTGATGAAGAAG GATCA-3', backward primer: 5'-ACTCCACAGGTGGGAA CAAG-3'.

Immunohistochemical staining of HCC tissue. The immunohistochemical studies were performed in formalin-fixed and paraffin-embedded $4-\mu \mathrm{m}$ thick tissue sections, using $\mathrm{K} 1$ autoantibody $(5 \mu \mathrm{g} / \mathrm{ml})$ and the streptavidin-biotin peroxidase complex method (3).

\section{Results}

B-cell hybridoma clones producing tumor-associated autoantibody generated from $\mathrm{H}$-ras $12 \mathrm{~V}$ transgenic HCC mouse model. To overcome the limitations of previous studies about tumor-associated autoantibodies, we intended to separate each tumor-associated autoantibody by separating each autoantibody-producing B cells, which was possible by construction of B cell hybridoma pool using splenocytes derived from HCC mouse model and clonal selection of B cell hybridoma which produce tumor-associated autoantibodies.

Wang and colleagues established H-ras transgenic mice using H-ras 12V gene under the control of the mouse albumin enhancer/promoter (3). Ras and related signal pathways play important roles in hepatocarcinogenesis (8). Ras/ERK upregulation is highest in human HCC with a poor prognosis and positively correlates with tumor proliferation, genomic instability, and microvascularization. Although the mutational activation of RAS protein occurs in hepatic tumors with an incidence of $5-58 \%(9,10)$, the receptor-mediated hyperactivation of RAS-dependent signal transduction pathway is a frequent event in human hepatocarcinogenesis $(11,12)$. Hepatic tumor formation in $\mathrm{H}$-ras transgenic mice showed a significant bias toward the males, which is consistent with the male prevalence of hepatic tumors in humans. Moreover, the MEK/ERK pathway is more strongly activated in male $\mathrm{H}$-ras $12 \mathrm{~V}$ transgenic mice than it is in females. These results indicate that the $\mathrm{H}$-ras $12 \mathrm{~V}$ transgenic mouse is a valuable animal model for use in studies on the molecular mechanisms associated with hepatocellular oncogenesis.

We obtained the splenocytes from these H-ras $12 \mathrm{~V}$ transgenic mice (nine male mice about 10 months old) and constructed B-cell hybridoma clones producing HCC-related autoantibodies without immunization of any other exogenous antigen or tumor cells. HCC formation was shown in seven mice (A, C, F , G, E, K and M), from which many more B-cell hybridoma clones were produced than the other two mice (B
A

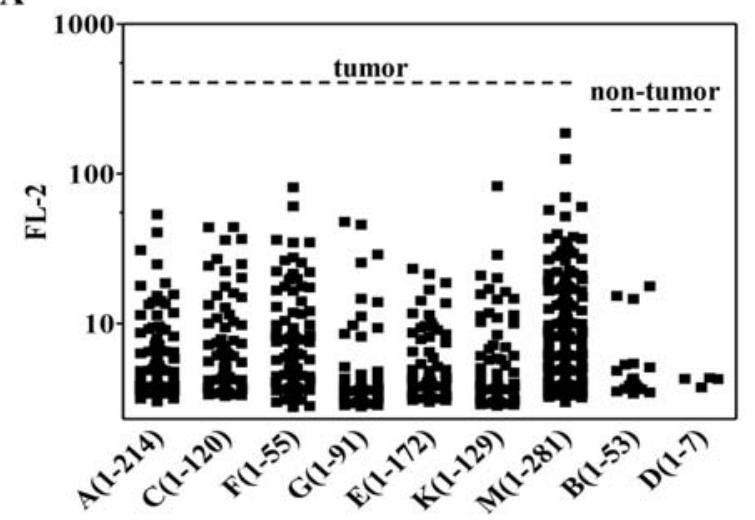

B

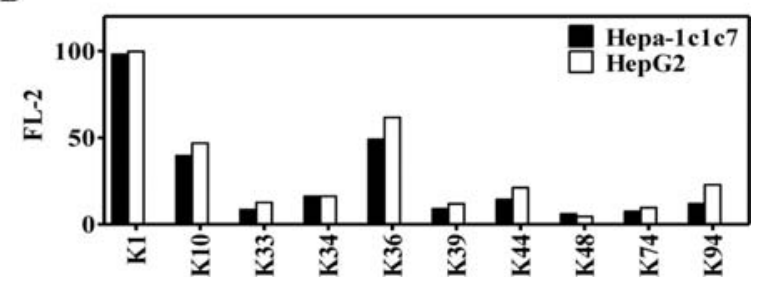

Figure 1. HCC-associated autoantibody, K1, was obtained from H-ras12V transgenic HCC mouse model. (A) Construction of autoantibody-generating B-cell hybridoma pool using splenocytes obtained from H-ras $12 \mathrm{~V} \mathrm{HCC}$ mouse model. Immortalized B-cell hybridomas were constructed and autoantibodies produced by these cells were examined by the reactivity against fixed and permeabilized HepG2 cells. Each spot reveals the reactivity of an individual autoantibody. (B) Among nine groups of autoantibody-generating B-cell hybridoma, $\mathrm{K}$ mouse-derived cells were analyzed first and the reactivity of $\mathrm{K} 1$ autoantibody was predominant.

and D) which did not develop HCC (Fig. 1A). Hundreds of autoantibodies generated by these B-cell hybridoma clones were examined separately by flow cytometric analysis of intracellular stained-tumor cells. The reactivity of autoantibodies which were generated from HCC-bearing mice was higher than that in the other two non-tumor mice, which indicates that generation of tumor-associated autoantibodies is correlated with the extent of tumorigenesis (Fig. 1A). B-cell hybridoma clones, which produce highly reactive autoantibodies, were selected for further studies.

For the first step of the characterization of these autoantibodies, the isotypes of about 40 autoantibodies were determined. Interestingly, most of the autoantibodies were $\operatorname{IgM}(27$ of 42) and the rest were $\operatorname{IgA}(11$ of 42$)$ or $\operatorname{IgG}$ (4 of 42 ), which may be related to the function or status of these antibodies in the process of tumorigenesis. We expected that the autoantibodies generated in the early stage of HCC formation would be more valuable as early biomarkers. Therefore, we started the analysis of autoantibodies from $\mathrm{K}$ mouse which had HCC in small size. Ten of the B-cell hybridoma clones from $\mathrm{K}$ mouse were selected and the reactivity of these autoantibodies was compared. As shown in Fig. 1B, the reactivity of $\mathrm{K} 1$ autoantibody was much higher than the other autoantibodies, which urged us to analyze it first.

K1 autoantigen expressed in tumor cells. The isotype of $\mathrm{K} 1$ autoantibody was IgM, which was purified from cell-cultured media or ascites fluid of K1 B cell hybridoma clone by protein 
A
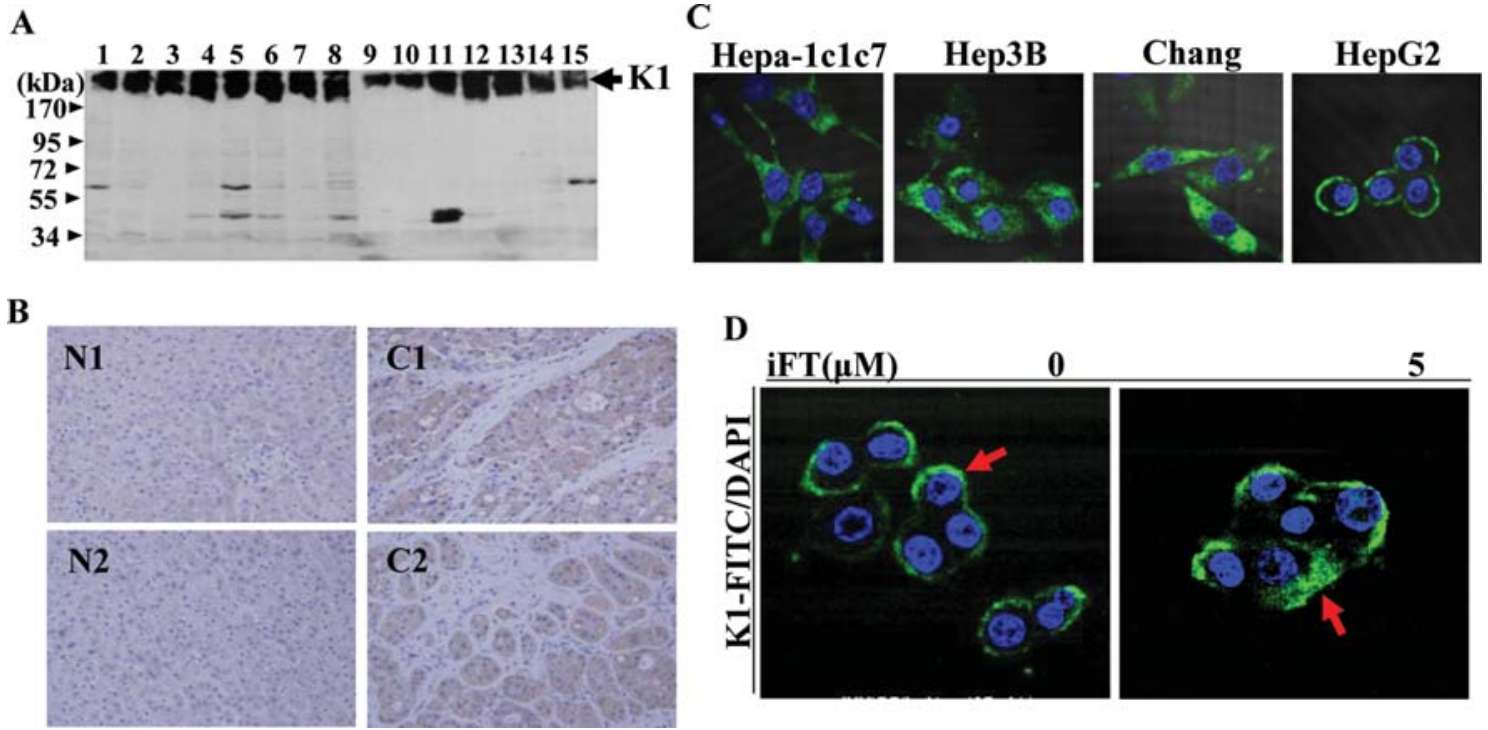

Figure 2. K1 autoantigen expressed in various tumor cells. (A) Western blot analysis on the target antigen of K1 autoantibody. Total cell lysates (50 $\mu$ g) were separated on 8-10\% SDS-PAGE gel, followed by blotting and probing with K1 autoantibody (1, Hepa1c1c7; 2, HepG2; 3, Hep3B; 4, Huh7; 5, PLC/PRF/5; 6, SK-Hep1; 7, Chang; 8, HeLa; 9, SNU601; 10, MCF7; 11, HT29; 12, A549; 15, HT22). (B) Immunohistochemical staining using K1 autoantibody in liver tissue from patients with HCC (C1 and C2) or normal subjects (N1 and N2). (C) Confocal microscopic analysis of intracellular localization of K1 autoantigen in liver cell lines (Hepa-1c1c7, Chang, Hep3B and HepG2). (D) Inhibition of Ras activity induced translocation of K1 autoantigen from plasma membrane to cytoplasm. Farensyltransferase inhibitor was treated on HepG2 cells for $16 \mathrm{~h}$ to a final concentration of $5 \mu \mathrm{M}$.

L-agarose. Using purified K1 autoantibody, target antigen was analyzed by several methods. As shown in Fig. 2A, K1 autoantibody was able to detect their target antigen by Western blotting. Its target antigen was a protein of a high molecular weight $(>200 \mathrm{kDa})$ and detected in various human tumor cells lines including hepatoma, colon cancer, and breast cancer, which means that its expression itself may not be specific to hepatoma.

To analyze the expression of $\mathrm{K} 1$ autoantigen in human hepatoma tissue, immunohistochemical staining using K1 autoantibody was performed. As shown in Fig. 2B, staining of $\mathrm{K} 1$ autoantibody was dominant in hepatoma tissue compared to normal. Four cases of hepatoma tissue were stained, of which two were strongly stained and others were stained slightly or similarly to that of normal liver, which might indicate heterogeneity of tumor cells.

Tumor-associated autoantigens, such as ECPKA, GRP78, and PRX, are cytosolic proteins overexpresssed in tumor cells (13-15). However, they are also detected in tumor patient sera (16-18), which induce humoral immune response. Although the mechanism of their secretion from tumor cells is not understood, their post-translational modification seems to be related to their secretion. During the process of secretion, autoantigens might stay in plasma membrane temporarily because tumor-associated antigens must translocate the plasma membrane for their secretion, which might be visualized by microscopic analysis of immunofluorescence-stained tumor cells. When intracellular localization of K1 antigen was analyzed by confocal microscopy, its expression was localized mainly in the cytoplasm of various liver cells (Hepa-1c1c7, Chang, Hep3B), with the exception of HepG2 cell, in which $\mathrm{K} 1$ antigen was incorporated in the plasma membrane (Fig. 2C). To examine whether the membrane localization of $\mathrm{K} 1$ autoantigen in HepG2 cells is dependent on the activity
Table I. Phage-displayed peptides obtained from biopanning with K1 autoantibody.

\begin{tabular}{ll}
\hline Clone number & Peptide Sequence $^{\mathrm{a}}$ \\
\hline K1-p3 & CRMSRRSNC \\
K1-p7 & CMRNRPKRC \\
K1-p22 & CRRRLNRTC \\
K1-p33 & CRMRIRRNC \\
K1-p34 & CHPHPRPRC \\
\hline
\end{tabular}

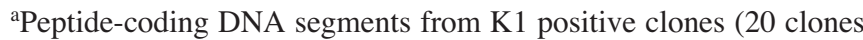
fron Cys-flanked 7-mer library) were sequenced and amino acid sequences were deduced. Five clones of different sequences were identified.

of Ras in tumor cells, K1 autoantigen was re-examined after treatment of a Ras inhibitor, a farensyltransferase inhibitor (19). As shown in Fig. 2D, treatment of a farensyltransferase inhibitor led K1 autoantigen to translocate from plasma membrane to cytoplasm, which might mean that a posttranslational modification on $\mathrm{K} 1$ antigen is related to ras activity, which cause temporary association of $\mathrm{K} 1$ antigen into plasma membrane.

Screening of mimotope against K1 antibody from the cyclic hepta-random peptide phage library. Autoantibody signature captured by using auto-antigenic mimotope-containing phages has been suggested as a potential diagnostic method for prostate cancer (20). Wang and his coworkers screened a phage display cDNA library derived from prostate cancer tissue with patient sera and used their selected mimotope 
A

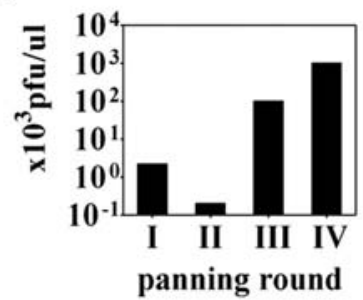

B

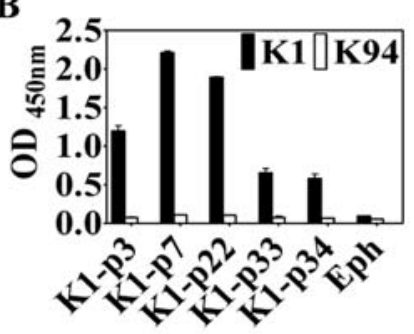

C

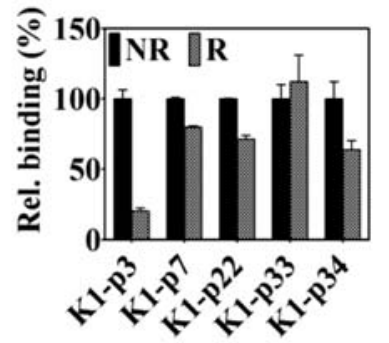

D

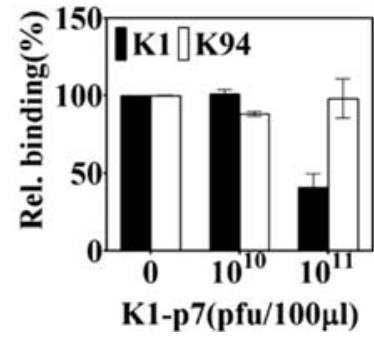

A

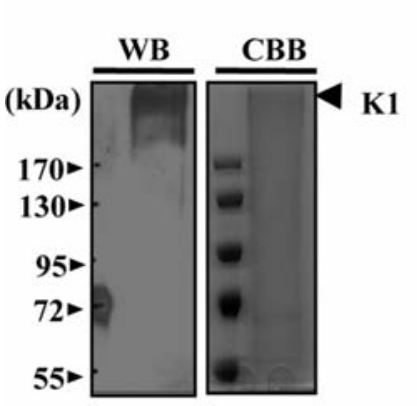

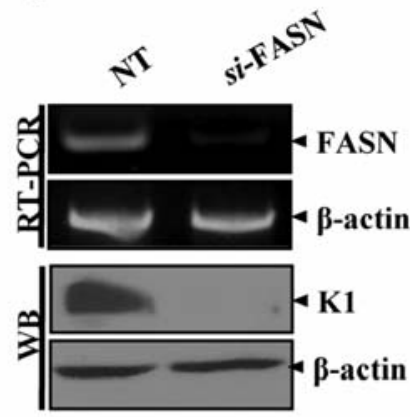

C

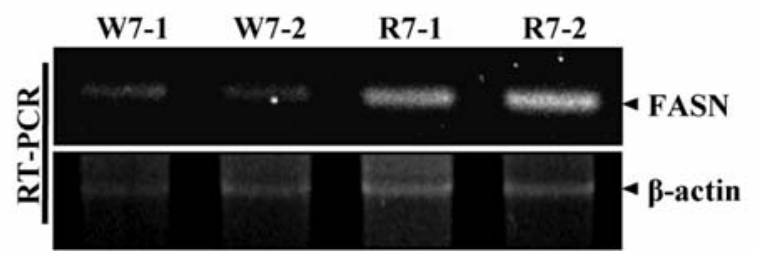

Fure 3. Panning of mimotope phage against K1 autoantibody. (A) For the selection of mimotope specific to $\mathrm{K} 1$ autoantibody, biopanning with $\mathrm{K} 1$ autoantibody was performed against random cyclic hepta-peptide phage library. (B) The reactivity of selected phages against K1 autoantibody was examined by phage ELISA. Empty phage (Eph) without an insert peptide sequence was used as control antigen and K94, another HCC-derived autoantibody, was used as control antibody. (C) Effect of reducing disulfide bonds on the reactivity of cyclic peptide mimotope. The cyclic mimotope phages, either untreated (NR) or treated with DTT and iodoacetamide (R), were tested for their reactivity against $\mathrm{K} 1$ autoantibody. The reactivity of treated phages $(\%)$ was calculated compared with untreated controls. (D) Competitive inhibition of $\mathrm{K} 1$ antibody binding to HepG2 cells with K1p7 mimotope phage. Fixed and permeabilized HepG2 cells were treated with K1 autoantibody, which was pre-adsorbed with or without K1p7 phages. The reactivity of phage-added reaction was calculated by comparing to the untreated control.

phages for tumor diagnosis successfully. Their results indicate that the antigenicity of autoantigens is restricted to one or two epitopes of a target protein, which makes it possible to detect specific autoantibodies with such defined antigenic structures, without using whole antigenic proteins. The restriction of antigenicity of a certain autoantigen to only one epitope was also shown in the case of GRP78 (17). In this study, we also tried to define antigenic sites against K1 autoantibody using the phage peptide library, which would be convenient 'bait' for the detection of autoantibody in patient sera. We screened the cyclic hepta-random peptide phage library, which is more effective to define the antigenic structure precisely (21). After four rounds of biopanning of the phage library (Fig. 3A), we obtained five phages with different insert peptide sequences (Table I), of which the reactivity against $\mathrm{K} 1$ antibody was analyzed by ELISA. As shown in Fig. 3B, K1 autoantibody was reactive to all of these mimotope phages, which have arginine-rich sequences. These phages confer hepta-peptide mimotope as a cyclic form maintained by a disulfide bond. To analyze the conformation-dependency of mimotopeantibody binding, mimotope phages of an intact form or reduced form were prepared and the reactivity was compared. As shown in Fig. 3C, K1 antibody binding was maintained at $>50 \%$ despite loosening of the cyclic conformation, which means that the binding of $\mathrm{K} 1$ antibody is partially dependent on the characteristics of mimotope peptide sequences. Among

Figure 4. Identification of K1 autoantigen as FASN. (A) Target antigen of K1 autoantibody was purified by using K1 autoantibody-conjugated beads. Purified protein fraction was Western blotted and immunostained with K1 autoantibody or stained with Coomassie Brilliant Blue. The protein band corresponding to K1 autoantigen was identified as FASN by mass spectrometric analysis. (B) Knockdown of FASN using siRNA eliminated the expression of K1 autoantibody-stained protein. Suppression of FASN was analyzed by RT-PCR and the expression of K1 autoantigen was analyzed by Western blotting. ß-actin was used as loading control. (C) RT-PCR analysis on the expression of FASN in liver tissue of H-ras12V HCC mouse model (R7-1 and R7-2). The normal liver tissue (W7-1 and W7-2) was used as a control.

seven selected phages, the reactivity of K1p7, having an insert peptide sequence of CMRNRPKRC, was highest and it competitively inhibit the binding of $\mathrm{K} 1$ antibody to HepG2 cells, which demonstrates that K1p7 phage properly mimics the epitope structure of a specific target antigen (Fig. 3D). Using the insert peptide sequence of $\mathrm{K} 1 \mathrm{p} 7$, a candidate protein was searched in protein sequence database, but no appropriate protein was found.

Protein identification of Kl autoantigen as FASN. For the identification of K1 antigen, immunoaffinity chromatography using $\mathrm{K} 1$ autoantibody-conjugated beads was performed. Hepa-1c1c7 cell lysate was used as a source of target antigen and specific elution was performed using a synthetic peptide of CMRNRPKRC, the mimotope sequence of K1p7 phage. The eluate was concentrated and separated on $8 \%$ SDSPAGE gel and the protein band corresponding to K1 antigen was excised, in-gel digested with trypsin. The peptide extracts of in-gel digestion were analyzed by Nano-LC-ESI-MS/MS (Fig. 4A; Table II) and, finally, K1 antigen was identified as fatty acid synthase (FASN), one of the well-known metabolic oncogene. To confirm the result of mass spectrometric analysis, HepG2 cells were transfected with siRNA against FASN and total cell lysates were analyzed by Western blotting. As shown in Fig. 4B, the protein band stained with $\mathrm{K} 1$ autoantibody in HepG2 cells disappeared when the expression of FASN was shut down. 
Table II. Identification of K1 autoantigen by mass spectrometric analysis.

\begin{tabular}{|c|c|c|c|c|c|}
\hline \multicolumn{2}{|c|}{ Gene_Symbol=Fasn } & \multirow{2}{*}{$\begin{array}{c}\text { Fatty acid synthase } \\
\text { Peptides }\end{array}$} & \multirow[t]{2}{*}{ Mass: 272257} & \multirow[t]{2}{*}{ Score: 80} & \multirow[t]{2}{*}{ Queries matched:4 } \\
\hline Query & & & & & \\
\hline 1 & R.L & QVADVTTSR.C & & 80 & 1069-1084 \\
\hline 2 & R.A & GCEAEAEAEALCFF & & 13 & $2345-2367$ \\
\hline 3 & K.L & PDGTCRSFDDSGSG & & 12 & $202-225$ \\
\hline 4 & R.C & DAHLPPGSMAAVGL & ECK.Q & 6 & $606-632$ \\
\hline
\end{tabular}

A

B

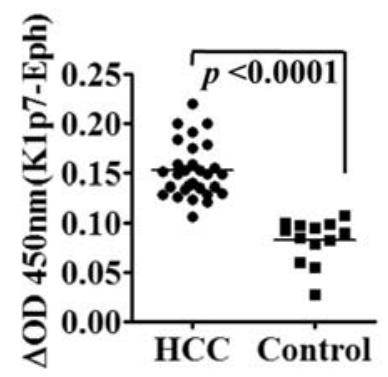

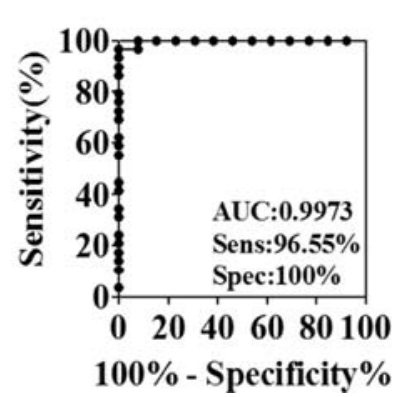

Figure 5. Detection of anti-FASN autoantibody in sera from patients with HCC. (A) Detection of autoantibody against FASN in HCC patient sera using K1p7 phage. The specific binding of autoantibody against K1p7 phage was expressed as the difference between the OD value of K1p7 ELISA and that of empty phage (Eph) ELISA. These experiments were repeated three times. (B) ROC curve of FASN-specific autoantibody ELISA in HCC patient and control sera.

FASN is a large $(\sim 265 \mathrm{kDa})$ homo-dimeric enzyme that is responsible for de novo fatty acid synthesis. Normal cells express low endogenous levels of FASN because they obtain significant amount of fatty acid from the diet. In contrast, FASN expression and activity in cancer cells can be extremely high because of increased requirements for long chain fatty acids (22). Also, as mentioned above, the presence of tumorassociated autoantibodies implicates the overexpression of target antigen in tumor tissue. Therefore, we examined the expression level of FASN in H-ras12V mouse model compared to normal subjects by RT-PCR. As shown in Fig. 4C, FASN expression in liver tissue from $\mathrm{H}$-ras $12 \mathrm{~V}$ mice was increased about 2-fold more than in controls. Collectively, these results suggest that FASN was overexpressed in H-ras-mediated hepatocarcinogenesis and induced a specific autoantibody.

Detection of autoantibodies against FASN in HCC patient sera. FASN is mainly expressed in normal hepatocytes and adipocytes, but it is active in cancer cells and the increased expression of FASN has emerged as a phenotype common to most human carcinomas, including colorectal, gastric, mammary, ovary, and prostate adenocarcinomas. Overexpression of FASN in tumor cells is often associated with a poor prognosis. FASN in cancer cells is uncoupled from dietary fat intake and variety of oncogenic changes [(amplification

of H-ras, erB-2, EGFR; (24)] result in FASN-catalyzed lipogenesis $(24,25)$. Also soluble FASN in blood is detected in the case of breast or prostate cancer, which is measured by ELISA for cancer diagnosis (26). But until now there were no reports on FASN-overexpression in HCC or tumor-related anti-FASN autoantibody.

Our results showed FASN was overexpressed in Rasmediated hepatocarcinogenesis and autoantibody against FASN was induced in HCC-bearing model mouse, which implicates that detection of soluble FASN or anti-FASN autoantibody can be used as a biomarker for HCC. In this study, we tried to setup a tumor-diagnostic method by measuring anti-FASN autoantibody in HCC patient sera, because tumor-associated autoantibodies are more efficient as tumor biomarkers than soluble tumor-associated proteins in sera, in the aspects of stability or simplicity (1). We developed indirect ELISA for the detection of autoantibody against FASN using the mimotope phage K1p7 as a coating antigen and human serum as autoantibody source. Human serum is a mixture of thousands of proteins that may induce non-specific antibody-antigen reactions (20). For the correction of the non-specific reaction, pre-adsorption of human sera was performed using ER2738 host cell extracts and empty phage. Empty phage that has no insert mimotope sequence was also used as a control coating antigen. On these conditions, 28 cases of HCC patient sera and 13 cases of normal sera were analyzed and the difference of OD value to K1p7 and empty phage was graphed. As shown in Fig. 5A, the reactivity of HCC patient sera against K1p7 phage was significantly different from that of sera from normal individuals $(\mathrm{p}<0.0001)$. The sensitivity of this ELISA was $96.55 \%$ and specificity was $100 \%$ when the cutoff value was 0.114 (AUC, 0.9973; Fig. 5B), which indicates that ELSA composed of K1p7 phage, a mimotope against anti-FASN autoantibody, can be used as an efficient diagnostic method of HCC.

\section{Discussion}

In this study, we suggest an anti-FASN autoantibody in patient sera as a novel HCC diagnostic marker. A diagnostic method for HCC was set up by using mimotope phage which could measure anti-FASN autoantibody in patient sera, of which sensitivity was $96.55 \%$ and specificity was $100 \%$. This is much more effective for HCC-diagnosis than any other methods measuring tumor-associated autoantibodies or serum markers (27). But, more extensive study with a large 
test group is still needed to confirm the utility of this method. Also, the association of anti-FASN autoantibody with other tumors or chronic liver disease must be examined.

Many studies exist on the oncogenic function of FASN, but little information has been appeared regarding extracellular FASN, its secretion mechanism, and induction of autoantibody. FASN in lipid raft was reported in prostate cancer cells (28). FASN is overexpressed in prostate cancer cells, and co-localized with Caveolin-1, a typical palmitoylated lipid-raft protein. FASN has a caveolin-binding motif sequence and co-localization of these proteins is dependent on activation of upstream signaling mediators. In our study, we also observed that FASN is membrane-associated in HepG2 cells and it is dependent on Ras activity. However, until now, the cause of membrane association of FASN was not clearly identified. In our opinion, FASN may be palmitoylated by oncogenic signals, which induces its membrane-association as well as secretion into serum. A study on the relationship between Ras activity and modification of FASN would be an interesting topic, which should be investigated in the future.

K1 HCC-associated autoantibody has been discovered from the B-cell hybridomas constructed from H-ras $12 \mathrm{~V}$ transgenic HCC mouse model. Although sera from patients with tumors are the ideal source for analyzing tumor-associated autoantibodies, a mouse tumor model is a good substitute source of tumor-associated autoantibodies. At first, much concern was voiced about the difference between patients with HCC and the mouse model, but results of analysis on $\mathrm{H}$-ras $12 \mathrm{~V}$ mice removed such worries. Now, hundreds of B-cell hybridoma clones, which were constructed from $\mathrm{H}$-ras $12 \mathrm{~V}$ transgenic mice, are available for the study of tumor-associated autoantibodies. We expect many more tumor marker autoantibodies will be found by studying these autoantibodies and the appropriate combination of these biomarkers could become a more efficient diagnostic method for tumors.

\section{Acknowledgements}

This study has been supported by the Korea Science and Engineering Foundation (KOSEF) grant (OGM2000811) and KRIBB research Initiative Program.

\section{References}

1. Cho-Chung YS: Autoantibody biomarkers in the detection of cancer (Review). Biochim Biophys Acta 762: 587-591, 2006.

2. Anderson KS and LaBaer J: The sentinel within: exploiting the immune system for cancer biomarkers (Review). J Proteome Res 4: 1123-1133, 2005.

3. Wang AG, Moon HB, Lee MR, Hwang CY, Kwon KS, Yu SL, Kim YS, Kim M, Kim JM, Kim SK, Lee TH, Moon EY, Lee DS and Yu DY: Gender-dependent hepatic alterations in H-ras12V transgenic mice. J Hepatol 43: 836-844, 2005.

4. Menendez JA and Lupu R: Fatty acid synthase and the lipogenic phenotype in cancer pathogenesis (Review). Nat Rev Cancer 7: 763-777, 2007

5. Park JH, Kim KL and Cho EW: Detection of surface asialoglycoprotein receptor expression in hepatic and extra-hepatic cells using a novel monoclonal antibody. Biotechnol Lett 28: 1061-1069, 2006.

6. Rojas G, Pupo A, Del Rosario Aleman M and Vispo NS: Preferential selection of Cys-constrained peptides from a random phage-displayed library by anti-glucitollysine antibodies. J Pept Sci 14: 1216-1221, 2008.
7. Lee JW, Lee SY, Song H and Yoo JS: The proteome of Mannheimia succiniciproducens, a capnophilic rumen bacterium. Proteomics 6: 3550-3566, 2006.

8. Hunter T: Oncoprotein networks. Cell 88: 333-346, 1997.

9. Bos JL: ras oncogenes in human cancer: a review. Cancer Res 49: 4682-4689, 1989

10. Kiba T, Tsuda H, Pairojkul C, Inoue S, Sugimura T and Hirohashi S: Mutations of the p53 tumor suppressor gene and the ras gene family in intrahepatic cholangiocellular carcinomas in Japan and Thailand. Mol Carcinog 8: 312-318, 1993.

11. Gotzmann J, Huber H, Thallinger C, Wolschek M, Jansen B, Schulte-Hermann R, Beug H and Mikulits W: Hepatocytes convert to a fibroblastoid phenotype through the cooperation of TGF-beta1 and Ha-Ras: steps towards invasiveness. J Cell Sci 115: 1189-1202, 2002.

12. Staib F, Hussain SP, Hofseth LJ, Wang XW and Harris CC: TP53 and liver carcinogenesis (Review). Hum Mutat 21: 201-216, 2003.

13. Cho YS, Park YG, Lee YN, Kim MK, Bates S, Tan L and ChoChung YS: Extracellular protein kinase A as a cancer biomarker: its expression by tumor cells and reversal by a myristate-lacking Calpha and RIIbeta subunit overexpression. Proc Natl Acad Sci USA 97: 835-840, 2000 .

14. Wang Q, He Z, Zhang J, Wang Y, Wang T, Tong S, Wang L, Wang S and Chen Y: Overexpression of endoplasmic reticulum molecular chaperone GRP94 and GRP78 in human lung cancer tissues and its significance. Cancer Detect Prev 29: 544-551, 2005.

15. Chang JW, Jeon HB, Lee JH, Yoo JS, Chun JS, Kim JH and Yoo YJ: Augmented expression of peroxiredoxin I in lung cancer. Biochem Biophys Res Commun 289: 507-512, 2001.

16. Nesterova MV, Johnson N, Cheadle C, Bates SE, Mani S, Stratakis CA, Khan IU, Gupta RK and Cho-Chung YS: Autoantibody cancer biomarker: extracellular protein kinase A. Cancer Res 66: 8971-8974, 2006.

17. Gonzalez-Gronow M, Cuchacovich M, Llanos C, Urzua C, Gawdi G and Pizzo SV: Prostate cancer cell proliferation in vitro is modulated by antibodies against glucose-regulated protein 78 isolated from patient serum. Cancer Res 66: 11424-11431, 2006.

18. Chang JW, Lee SH, Jeong JY, Chae HZ, Kim YC, Park ZY and Yoo YJ: Peroxiredoxin-I is an autoimmunogenic tumor antigen in non-small cell lung cancer. FEBS Lett 579: 2873-2877, 2005.

19. Doll RJ, Kirschmeier P and Bishop WR: Farnesyltransferase inhibitors as anticancer agents: critical crossroads (Review). Curr Opin Drug Discov Devel 7: 478-486, 2004.

20. Wang X, Yu J, Sreekumar A, Varambally S, Shen R, Giacherio D, Mehra R, Montie JE, Pienta KJ, Sanda MG, Kantoff PW, Rubin MA, Wei JT, Ghosh D and Chinnaiyan AM: Auto-antibody signatures in prostate cancer. N Engl J Med 353: 1224-1235, 2005.

21. Hoess RH, Mack AJ, Walton H and Reilly TM: Identification of a structural epitope by using a peptide library displayed on filamentous bacteriophage. J Immunol 153: 724-729, 1994.

22. Kuhajda FP: Fatty acid synthase and cancer: new application of an old pathway (Review). Cancer Res 66: 5977-5980, 2006.

23. Weng MS, Ho CT, Ho YS and Lin JK: The anaphthoquinone inhibits fatty acid synthase expression in EGF-stimulated human breast cancer cells via the regulation of EGFR/ErbB-2 signaling. Toxicol Appl Pharmacol 218: 107-118, 2007.

24. Van de Sande T, De Schrijver E, Heyns W, Verhoeven G and Swinnen JV: Role of the phosphatidylinositol 3'-kinase/PTEN/ Akt kinase pathway in the overexpression of fatty acid synthase in LNCaP prostate cancer cells. Cancer Res 62: 642-646, 2002.

25. Porstmann T, Griffiths B, Chung YL, Delpuech O, Griffiths JR, Downward J and Schulze A: PKB/Akt induces transcription of enzymes involved in cholesterol and fatty acid biosynthesis via activation of SREBP. Oncogene 24: 6465-6481, 2005.

26. Wang Y, Kuhajda FP, Sokoll LJ and Chan DW: Two-site ELISA for the quantitative determination of fatty acid synthase. Clin Chim Acta 304: 107-115, 2001.

27. Gomaa AI, Khan SA, Leen EL, Waked I and Taylor-Robinson SD: Diagnosis of hepatocellular carcinoma (Review). World J Gastroenterol 15: 1301-1314, 2009.

28. Di Vizio D, Adam RM, Kim J, Kim R, Sotgia F, Williams T, Demichelis F, Solomon KR, Loda M, Rubin MA, Lisanti MP and Freeman MR: Caveolin-1 interacts with a lipid raft-associated population of fatty acid synthase. Cell Cycle 7: 2257-2267, 2008. 